distribution. These characteristics of carbon led to better filling of carbon into the zeolite nanochannels, which are shown by nuclear magnetic resonance analysis.

Since the highly ordered structure suggested the absence of mesoporosity, the researchers concluded that one possible carbon structure may be "a curved graphene structure...accommodated to the curved inner surface of the zeolite nanochannels."

KINSON C. KAM

\section{Theories of Polymer Crystallization Challenged by Molecular Simulations}

A team of researchers at the University of Massachusetts has demonstrated their modeling results on polymer crystallization from solution in which entropic barriers control the initial lamellar thickness by initiation of crystal nuclei. The nuclei then grow by chain absorption at the crystalline interface, and the lamellae thicken in a cooperative process requiring mobility of all chains in the crystal. These results challenge the conventional Lauritzen-Hoffman $(\mathrm{LH})$ theory and its generalizations.

In contrast to the crystallization of small molecules, the crystallization of polymers from solution is still poorly understood. Polymer molecules can participate in different initial nuclei, which leads to entropic frustration and incomplete crystallization, in which the polymer chains fold back and forth to form crystalline lamellae. So far, thermodynamics estimates have failed to predict the initial lamellar thickness, and all estimates are about 2 orders of magnitude higher than the $10 \mathrm{~nm}$ observed. There is also some controversy about the growth mechanism of the lamellae. As reported in the November 19, 2001, issue of Physical Review Letters, the group used Langevin dynamics simulations to reinvestigate these problems.

The results of the dynamics simulations show that the initial crystallization from solution does not occur by spinodal dynamics, but through a nucleation and growth mechanism, in which the initial lamellar thickness is dictated by freeenergy barriers. The initial thickness is spontaneously selected, and the chains thicken by negotiating free-energy barriers before asymptotically approaching a thickness that is much smaller than the thermodynamically predicted extended chain limit. Chain growth occurs by simultaneous adsorption and crystallographic registry of diffusing chains at the growth front. In contrast to the LH model, this step is not hindered by an energy barrier. The chains then rearrange to form stems that are commensurate with the crystal thickness at the growth front.

M. Muthukumar, Barrett Professor in the Department of Polymer Science and Engineering, said, "Our results suggest that the definitions of quench depth and equilibrium melting temperature, which depend on the equilibrium lamellar thickness, need to be redefined. This opens up 40 years of accumulated data for re-analysis."

CORA LIND

\section{Hypersensitization Improves Performance of Rare-Earth-Doped Active Waveguides}

The photosensitivity of optical waveguides has been enhanced in the past with the addition of hydrogen. The price for such sensitivity-enhancement includes thermal instability, additional absorption due to hydrogen-induced bands, lower gain, and out-diffusion issues during device fabrication. J. Canning and K. Sommer of the Optical Fibre Technology Centre at the University of Sydney have resolved these issues by using hydrogen hypersensitization in active optical waveguides.

Typically, waveguides are patterned by UV irradiation. To effectively demonstrate the advantages of hypersensitized fibers as compared with untreated and hydrogen-loaded fibers, a total of six specimens were measured for small signal gain and lifetime according to the following plan, as reported in the December 1, 2001, issue of Optics Letters. Two different types of fibers were used in the experiments. The first batch, consisting of three samples, was $12 \%$ germanosilicate fibers doped with $0.1 \mathrm{~mol} \% \mathrm{Er}^{3+}$. The second batch, consisting also of three samples, was $17 \%$ phosphosilicate fibers doped with $0.3 \mathrm{~mol} \%$ and $3 \mathrm{~mol} \%$ of $\mathrm{Er}^{3+}$ and $\mathrm{Yb}^{3+}$, respectively. In addition, three preirradiation treatments were applied to the samples. Two samples, one from each batch, were not modified. Two samplesagain, one from each batch-were fully loaded with hydrogen at $200 \mathrm{~atm}, 80^{\circ} \mathrm{C}$, for four days. The remaining two samples were hypersensitized samples; that is, hydrogen was introduced and the samples were given a preliminary exposure before the hydrogen was allowed to out-diffuse. Gratings in the fibers were subsequently written with a 244-nm (germanosilicate fibers) and a 193-nm (phosphosilicate) UV laser. The small-signal gain coefficient was measured using a tunable laser and a 980-nm pump. A chopper and an oscilloscope were used to measure the excited-state lifetime.

In the germanosilicate samples, small signal gain was reduced with the intro- duction of hydrogen throughout the 1520-1560-nm range, according to the researchers. They reported that the hypersensitized sample fared better than the fully loaded samples. Hydrogen caused little deviation in the upper excited-state lifetime, they said.

Small signal gain in the hypersensitized phosphosilicate fiber was comparable to that of the unmodified fiber. Lifetime in the hypersensitized fiber was slightly lower than that of the untreated fiber. The fully loaded fiber suffered significantly in both signal attenuation and excited-state lifetime.

The researchers concluded from this set of experiments that hypersensitization is a readily available method for improving stability, reliability, and efficiency of optical fibers and waveguides.

JUNE LAU

\section{Molecules Designed to Mimic Bone at the Nanolevel}

A research team at Northwestern University led by Samuel I. Stupp, the Board of Trustees Professor of Materials Science, Chemistry, and Medicine, has designed molecules that self-assemble into a three-dimensional structure that mimics the key features of human bone at the nanoscale level. These features include collagen nanofibers that promote mineralization and mineral nanocrystals. Collagen is found in most human tissues, including the heart, the eye, blood vessels, skin, cartilage, and bone, and gives these tissues their structural strength.

As reported in the November 23, 2001, issue of Science, the researchers created self-assembled nanofibers $(\sim 8 \mathrm{~nm}$ in diameter) that resemble the collagen fibrils of real bone in shape and size. When the nanofibers were exposed to solutions containing calcium and phosphate ions, the fibers became covered with hydroxyapatite crystals. These thin, rectangular mineral wafers grew on the nanofibers in a direction parallel to the fiber's length, mimicking hydroxyapatite-crystal growth on collagen in the formation of real bone.

According to the researchers, the assembly of the nanofibers themselves can be reversed by changing the $\mathrm{pH}$ level of the fibers' environment. The fibers also can be polymerized or cross-linked by oxidation to give them additional strength, a process that also can be reversed. The researchers said that the versatility of the nanofiber system offers the possibility of using the organic fibers as "cargo carriers," possibly for drug delivery to a specific point in the body. Natural enzymes found in the body can disassemble the fibers so that their cargo can be released. 